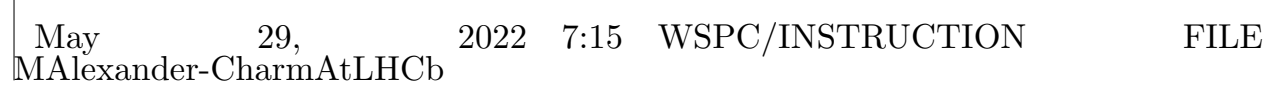

International Journal of Modern Physics: Conference Series

(c) World Scientific Publishing Company

\title{
CHARM: MIXING, CP VIOLATION AND RARE DECAYS AT LHCB
}

\author{
MICHAEL ALEXANDER* \\ Physics 8 Astronomy, University of Glasgow, \\ Glasgow, G12 8QQ, United Kingdom \\ michael.alexander@glasgow.ac.uk
}

On behalf of the $\mathrm{LHCb}$ collaboration

Received $30^{\text {th }}$ November 2013

Revised $30^{\text {th }}$ November 2013

\begin{abstract}
Recent results on mixing, $\mathrm{CP}$ violation and rare decays in charm physics from the $\mathrm{LHCb}$ experiment are presented. Study of "wrong-sign" $\mathrm{D}^{0} \rightarrow \mathrm{K}^{+} \pi^{-}$decays provides the highest precision measurements to date of the mixing parameters $x^{\prime 2}$ and $y^{\prime}$, and of CP violation in this decay mode. Direct and indirect $\mathrm{CP}$ violation in the $\mathrm{D}^{0}$ system are probed to a sensitivity of around $10^{-3}$ using $\mathrm{D}^{0} \rightarrow \mathrm{K}^{+} \mathrm{K}^{-}$and $\mathrm{D}^{0} \rightarrow \pi^{+} \pi^{-}$decays and found to be consistent with zero. Searches for the rare decays $\mathrm{D}_{(s)}^{+} \rightarrow \pi^{+} \mu^{+} \mu^{-}$, $\mathrm{D}_{(s)}^{+} \rightarrow \pi^{-} \mu^{+} \mu^{+}$and $\mathrm{D}^{0} \rightarrow \mu^{+} \mu^{-}$find no evidence of signal, but set the best limits on branching fractions to date. Thus, despite many excellent results in charm physics from $\mathrm{LHCb}$, no evidence for physics beyond the Standard Model is found.

Keywords: LHC, LHCb, charm, CP violation, mixing, rare decays

PACS numbers:13.25.Ft, 14.40.Lb
\end{abstract}

\section{Introduction}

$\mathrm{CP}$ violation and the branching fractions of rare decays in charm physics can be significantly enhanced beyond Standard Model (SM) predictions by the presence of non-SM particles. Precision measurements of these parameters can thus provide evidence for, or strict limits on, new physics.

The LHCb detector ${ }^{1}$ at the LHC is specifically designed for precision measurements of $\mathrm{CP}$ violation in decays involving $c$ and $\mathrm{b}$ quarks. The Vertex Locator provides fine tracking around the interaction point, achieving decay-time resolutions of around 50 fs for $\mathrm{D}^{0}$ mesons; the full tracking system yields momentum resolutions of $\sigma(p) / p \sim 0.5 \%$; and the two Ring Imaging Cherenkov detectors provide clean separation of pions and kaons. Additionally, the $c \bar{c}$ production cross section in the collisions provided by the LHC is very large. With $1.1 \mathrm{fb}^{-1}$ integrated luminosity

*232b Kelvin Building, University of Glasgow, Glasgow, UK. 
recorded at $\sqrt{s}=7 \mathrm{TeV}$ in 2011 and $2.1 \mathrm{fb}^{-1}$ at $\sqrt{s}=8 \mathrm{TeV}$ in 2012 this makes $\mathrm{LHCb}$ an excellent source of high statistics datasets for the study of charm physics.

\section{Mixing and CP Violation in $\mathbf{D}^{0} \rightarrow \mathrm{K}^{ \pm} \pi^{\mp}$ Decays}

The "right-sign" (RS) decay of $\mathrm{D}^{0} \rightarrow \mathrm{K}^{-} \pi^{+\mathrm{a}}$ occurs predominantly through a Cabibbo-favored $(\mathrm{CF})$ process in which no $\mathrm{D}^{0}-\overline{\mathrm{D}}^{0}$ mixing occurs. The "wrong-sign" (WS) $\mathrm{D}^{0} \rightarrow \mathrm{K}^{+} \pi^{-}$decay occurs with roughly equal rate via a doubly-Cabibbosuppressed (DCS) process and one in which the $\mathrm{D}^{0}$ meson first mixes and then decays via the $\mathrm{CF}$ process. The ratio of the decay rate of WS decays to that of RS decays as a function of the decay time of the $\mathrm{D}^{0}$ meson is thus sensitive to the mixing parameters $x^{\prime 2}$ and $y^{\prime}$ and is given by

$$
R(t)=\frac{N_{W S}(t)}{N_{R S}(t)}=R_{D}+\sqrt{R_{D}} y^{\prime} t+\frac{x^{\prime 2}+y^{\prime 2}}{4} t^{2},
$$

where

$$
\begin{gathered}
R_{D}=\left|\frac{A_{D C S}}{A_{C F}}\right|^{2}, x^{\prime}=x \cos (\delta)+y \sin (\delta), y^{\prime}=-x \sin (\delta)+y \cos (\delta), \\
\delta=\arg \left(\frac{A_{D C S}}{A_{C F}}\right), x=\frac{\Delta m_{\mathrm{D}^{0}}}{\Gamma_{\mathrm{D}^{0}}}, y=\frac{\Delta \Gamma_{\mathrm{D}^{0}}}{2 \Gamma_{\mathrm{D}^{0}}},
\end{gathered}
$$

$A_{D C S(C F)}$ is the amplitude of the DCS (CF) decay, $\Delta m_{\mathrm{D}^{0}}$ is the mass difference of the mass eigenstates of the $\mathrm{D}^{0}$ system (defined as $\left|\mathrm{D}_{H, L}^{0}\right\rangle=p\left|\mathrm{D}^{0}\right\rangle \pm q\left|\overline{\mathrm{D}}^{0}\right\rangle$ ), $\Delta \Gamma_{\mathrm{D}^{0}}$ the decay width difference, and $\Gamma_{\mathrm{D}^{0}}$ the decay width of the $\mathrm{D}^{0}$ meson. Any discrepancy in $R(t)$ between initial states of $\mathrm{D}^{0}$ and $\overline{\mathrm{D}}^{0}$ would indicate $\mathrm{CP}$ violation.

$R(t)$ is measured by firstly reconstructing and selecting $\mathrm{D}^{*+} \rightarrow \mathrm{D}^{0} \pi_{s}^{+}$candidates with $\mathrm{D}^{0} \rightarrow \mathrm{K}^{ \pm} \pi^{\mp}{ }^{2}$. The charge of the $\pi_{s}^{+}$track gives the flavor of the $\mathrm{D}^{0}$ candidate at production. Backgrounds, predominantly from $\mathrm{D}^{0}$ decays associated with a random $\pi_{s}^{+}$track, are distinguished from signal by the distribution of the invariant mass of the $\mathrm{D}^{*+}$ candidates $\left(m_{\mathrm{D}^{*+}}\right)$. An additional background arises from $\mathrm{D}^{0}$ mesons produced in decays of $\mathrm{B} \rightarrow \mathrm{D}^{0} X$. These are strongly suppressed by a cut on the impact parameter (IP) $\chi^{2}$ of the $\mathrm{D}^{0}$ candidate and a systematic uncertainty assigned to any remaining contribution. WS and RS candidates are divided into bins of $\mathrm{D}^{0}$ decay time and the distribution of $m_{\mathrm{D}^{*+}}$ fitted in each bin to obtain the yields. The ratio of WS to RS yields is then plotted against decay time to give $R(t)$ and fitted with Eq. 2 to determine $R_{D}, x^{\prime 2}$ and $y^{\prime}$.

The results of fits for $x^{\prime 2}$ and $y^{\prime}$ from the full 2011 and 2012 datasets are shown in Fig. 1. The cases in which $\mathrm{CP}$ violation is allowed, only indirect $\mathrm{CP}$ violation is allowed, and no $\mathrm{CP}$ violation is allowed are all considered. The $\mathrm{CP}$ conservation case gives

$$
R_{D}=(3.568 \pm 0.066) \times 10^{-3}, x^{2}=(5.5 \pm 4.9) \times 10^{-5}, y^{\prime}=(4.8 \pm 1.0) \times 10^{-3},
$$

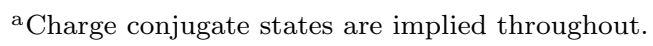




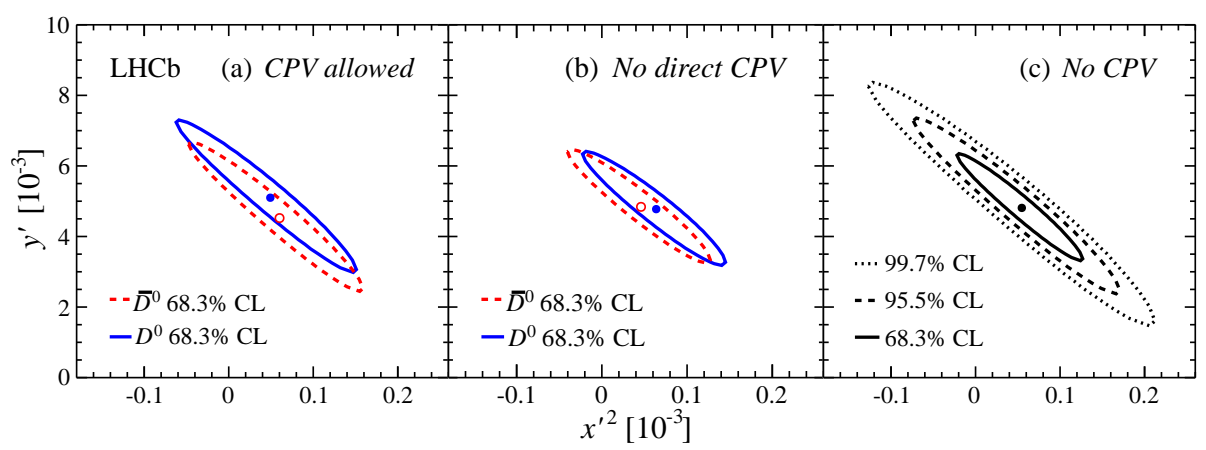

Fig. 1. The results of fits for $x^{\prime 2}$ and $y^{\prime}$ in the case that (left) CP violation is allowed, (middle) only indirect $\mathrm{CP}$ violation is allowed, and (right) no $\mathrm{CP}$ violation is allowed.

which excludes the no mixing hypothesis at $>10 \sigma$. Additionally, the cases for which $\mathrm{CP}$ violation is allowed find

$$
\frac{R_{D}\left(\mathrm{D}^{0}\right)-R_{D}\left(\overline{\mathrm{D}}^{0}\right)}{R_{D}\left(\mathrm{D}^{0}\right)+R_{D}\left(\overline{\mathrm{D}}^{0}\right)}=(-0.7 \pm 1.9) \%,
$$

and $0.75<|q / p|<1.24$ at $68.3 \%$ confidence. These are the most precise single measurements of these parameters to date and show no evidence for $\mathrm{CP}$ violation.

\section{Indirect CP Violation in $\mathbf{D}^{0} \rightarrow \mathbf{h}^{+} \mathbf{h}^{-}$Decays}

The parameter $A_{\Gamma}$, defined below, gives access primarily to indirect $\mathrm{CP}$ violation as

$$
A_{\Gamma}=\frac{\tau_{\text {eff }}\left(\overline{\mathrm{D}}^{0} \rightarrow \mathrm{h}^{+} \mathrm{h}^{-}\right)-\tau_{\text {eff }}\left(\mathrm{D}^{0} \rightarrow \mathrm{h}^{+} \mathrm{h}^{-}\right)}{\tau_{\text {eff }}\left(\overline{\mathrm{D}}^{0} \rightarrow \mathrm{h}^{+} \mathrm{h}^{-}\right)+\tau_{\text {eff }}\left(\mathrm{D}^{0} \rightarrow \mathrm{h}^{+} \mathrm{h}^{-}\right)} \simeq\left[\frac{1}{2}\left(A_{m}+A_{d}\right) y \cos \phi-x \sin \phi\right],
$$

where $\tau_{\text {eff }}$ is the effective lifetime, $\mathrm{h}$ can be either a pion or kaon, and

$$
A_{m}=\frac{|q / p|^{2}-|p / q|^{2}}{|q / p|^{2}+|p / q|^{2}}, A_{d}=\frac{\left|A_{f} / \bar{A}_{f}\right|^{2}-\left|\bar{A}_{f} / A_{f}\right|^{2}}{\left|A_{f} / \bar{A}_{f}\right|^{2}+\left|\bar{A}_{f} / A_{f}\right|^{2}}, \phi=\arg \left(\frac{q}{p} \frac{\bar{A}_{f}}{A_{f}}\right),
$$

with $A_{f}\left(\bar{A}_{f}\right)$ the amplitude of the $\mathrm{D}^{0}\left(\overline{\mathrm{D}}^{0}\right)$ meson decaying to the given final state.

Similarly to the analysis method discussed in Sec. 2 the decay chain $\mathrm{D}^{*+} \rightarrow \mathrm{D}^{0} \pi_{s}^{+}$ is used to obtain the flavor of the $\mathrm{D}^{0}$ candidate at production ${ }^{3}$. The $\mathrm{K}^{+} \mathrm{K}^{-}$and $\pi^{+} \pi^{-}$ final states are both analyzed. For the $\mathrm{K}^{+} \mathrm{K}^{-}$final state backgrounds from partially reconstructed three-body $\mathrm{D}^{0}$ decays contribute in addition to combinatorics. These are distinguished by a fit to the distribution of $m_{\mathrm{D}^{0}}$ and $\Delta m \equiv m_{\mathrm{D}^{*+}}-m_{\mathrm{D}^{0}}$.

The effective lifetime is obtained by performing an unbinned maximum likelihood fit to the decay-time distribution using a data-driven, per-candidate method to correct for the biasing effect of the candidate selection ${ }^{4}$. Additionally, the distribution of the IP $\chi^{2}$ of the $\mathrm{D}^{0}$ candidates is used to distinguish the background from $\mathrm{B} \rightarrow \mathrm{D}^{0} X$ decays. Examples of fits to the $\mathrm{D}^{0}$ IP $\chi^{2}$ and decay-time distributions are shown in Fig. 2. 

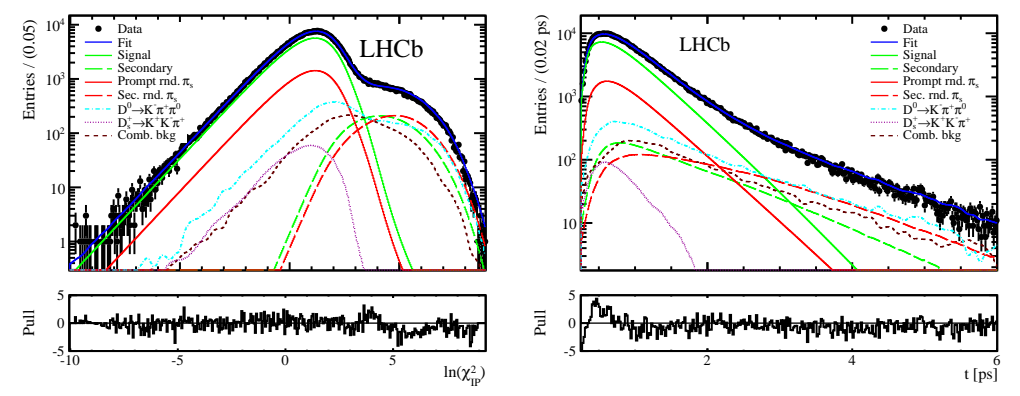

Fig. 2. Examples of fits to the impact parameter $\chi^{2}$ and decay-time distributions for the $\mathrm{K}^{+} \mathrm{K}^{-}$ final state.

From the 2011 dataset these fits give

$$
A_{\Gamma}^{K K}=(-0.35 \pm 0.62 \pm 0.12) \times 10^{-3}, A_{\Gamma}^{\pi \pi}=(0.33 \pm 1.03 \pm 0.14) \times 10^{-3},
$$

where dominant systematics arise from the accuracy of the acceptance correction and the modeling of the backgrounds. Thus, no evidence for indirect $\mathrm{CP}$ violation is found.

\section{Direct CP Violation in $\mathbf{D}^{0} \rightarrow \mathbf{h}^{+} \mathbf{h}^{-}$Decays}

The parameter

$$
\Delta \mathcal{A}^{\mathrm{CP}}=\mathcal{A}^{\mathrm{CP}}\left(\mathrm{D}^{0} \rightarrow \mathrm{K}^{+} \mathrm{K}^{-}\right)-\mathcal{A}^{\mathrm{CP}}\left(\mathrm{D}^{0} \rightarrow \pi^{+} \pi^{-}\right),
$$

where $\mathcal{A}^{\mathrm{CP}}$ is the time-integrated $\mathrm{CP}$ asymmetry of the given decay, gives direct access to direct $\mathrm{CP}$ violation as the production asymmetry of the $\mathrm{D}^{0}$ meson cancels in the difference. To ensure full cancellation of production and detection asymmetries between the $\mathrm{K}^{+} \mathrm{K}^{-}$and $\pi^{+} \pi^{-}$final states the $\mathrm{K}^{+} \mathrm{K}^{-}$candidates are weighted so that their kinematic distributions match those of $\pi^{+} \pi^{-}$candidates.

Two independent datasets are used to measure $\Delta \mathcal{A}^{\mathrm{CP}}$ : one in which the flavor of the $\mathrm{D}^{0}$ candidate at production is determined using $\mathrm{D}^{*+} \rightarrow \mathrm{D}^{0} \pi_{s}^{+}$decays ${ }^{5}$, comprising predominantly prompt $\mathrm{D}^{0}$ decays, and one in which the decay $\mathrm{B}^{-} \rightarrow \mathrm{D}^{0} \mu^{-}$is reconstructed, with the charge of the $\mu^{-}$giving the flavor of the $\mathrm{D}^{0}$ candidate ${ }^{6}$. The yields are determined by fits to the $\Delta m$ distribution for the pion tagged sample, and $m_{\mathrm{D}^{0}}$ for the muon tagged sample.

Using the 2011 dataset the measurements obtained are

$$
\Delta \mathcal{A}^{\mathrm{CP}}(\pi \text { tagged })=-0.34 \pm 0.15 \pm 0.10 \%, \Delta \mathcal{A}^{\mathrm{CP}}(\mu \text { tagged })=+0.49 \pm 0.30 \pm 0.14 \%,
$$

where the $\pi$ tagged measurement is preliminary. This gives an average of

$$
\Delta \mathcal{A}^{\mathrm{CP}}=-0.15 \pm 0.16 \% .
$$

Thus no evidence of direct $\mathrm{CP}$ violation is found. 

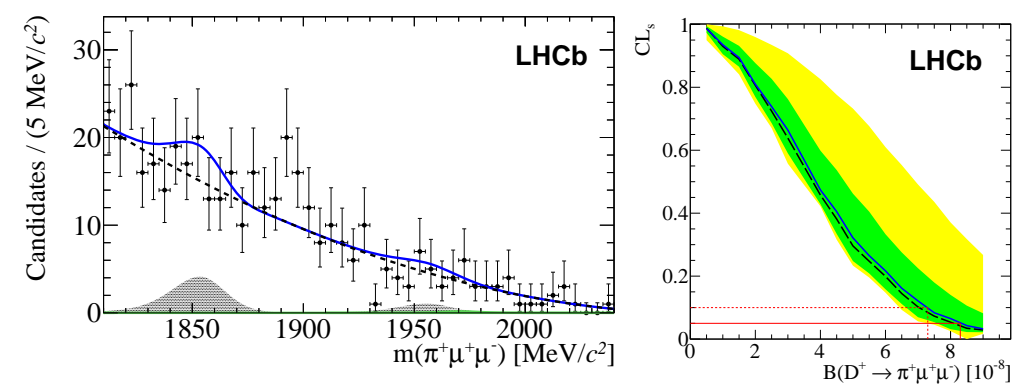

Fig. 3. (Left) the fit to the $\pi^{+} \mu^{+} \mu^{-}$invariant mass distribution in the low $\mu^{+} \mu^{-}$mass region, with background from $\mathrm{D}^{+} \rightarrow \pi^{+} \pi^{+} \pi^{-}$shown in solid gray; and (right) the confidence limits for $\mathcal{B}\left(\mathrm{D}^{+} \rightarrow \pi^{+} \mu^{+} \mu^{-}\right)$.

\section{Rare Decays}

The search for decays of $\mathrm{D}_{(s)}^{+} \rightarrow \pi^{+} \mu^{+} \mu^{-}$is sensitive to new physics as the process $c \rightarrow \mathrm{u} \mu^{+} \mu^{-}$is predicted to have a branching fraction of $\mathcal{O}\left(10^{-9}\right)$ in the $\mathrm{SM}^{7}$. Similarly, the lepton number violating decays $\mathrm{D}_{(s)}^{+} \rightarrow \pi^{-} \mu^{+} \mu^{+}$are forbidden in the $\mathrm{SM}$ but can occur in some new physics scenarios, e.g. with the existence of Majorana neutrinos.

The distributions of $\pi \mu \mu$ invariant mass are fitted in bins of $\mu^{+} \mu^{-}\left(\pi^{-} \mu^{+}\right)$ invariant mass in order to determine the yields ${ }^{8}$. The limits on the signal yields are normalized to the yields found in the $m_{\mu^{+} \mu^{-}}$region containing the $\phi$ resonance and combined with the well known $\mathcal{B}\left(\mathrm{D}_{(s)}^{+} \rightarrow \pi^{+} \phi\left(\rightarrow \mu^{+} \mu^{-}\right)\right)$to obtain limits on the partial branching fractions of $\mathrm{D}_{(s)}^{+} \rightarrow \pi^{ \pm} \mu^{+} \mu^{\mp}$ in each bin of $m_{\mu^{+} \mu^{-}}\left(m_{\pi^{-} \mu^{+}}\right)$. The total branching fractions of non-resonant decays are also constrained by combining information in bins of $m_{\mu^{+} \mu^{-}}\left(m_{\pi^{-} \mu^{+}}\right)$, assuming a phase-space model. An example of a mass fit and branching fraction confidence limits for $\mathrm{D}^{+} \rightarrow \pi^{+} \mu^{+} \mu^{-}$decays are shown in Fig. 3.

Using the 2011 dataset the resulting $90 \%$ confidence limits on the total nonresonant branching fractions are

$$
\begin{array}{ll}
\mathcal{B}\left(\mathrm{D}^{+} \rightarrow \pi^{+} \mu^{+} \mu^{-}\right)<7.3 \times 10^{-8}, & \mathcal{B}\left(\mathrm{D}_{s}^{+} \rightarrow \pi^{+} \mu^{+} \mu^{-}\right)<4.1 \times 10^{-7}, \\
\mathcal{B}\left(\mathrm{D}^{+} \rightarrow \pi^{-} \mu^{+} \mu^{+}\right)<2.2 \times 10^{-8}, & \mathcal{B}\left(\mathrm{D}_{s}^{+} \rightarrow \pi^{-} \mu^{+} \mu^{+}\right)<1.2 \times 10^{-7},
\end{array}
$$

which give a factor of roughly $10^{-2}$ improvement over the previous best limits.

A similar search for $\mathrm{D}^{0} \rightarrow \mu^{+} \mu^{-}$decays using the 2011 dataset finds $\mathcal{B}\left(\mathrm{D}^{0} \rightarrow \mu^{+} \mu^{-}\right)<6.2 \times 10^{-9}$ at $90 \%$ confidence $^{9}$. Thus, no evidence for new physics has yet been found via rare decays.

\section{Conclusions}

Recent results on mixing, $\mathrm{CP}$ violation and rare decays in charm physics from the $\mathrm{LHCb}$ experiment were presented. Study of $\mathrm{D}^{0} \rightarrow \mathrm{K}^{+} \pi^{-}$decays has provided the highest precision measurements of the mixing parameters $x^{\prime 2}$ and $y^{\prime}$ to date, and 
of $\mathrm{CP}$ violation in this decay mode. Direct and indirect $\mathrm{CP}$ violation in the $\mathrm{D}^{0}$ system have been probed to a sensitivity of around $10^{-3}$ using $\mathrm{D}^{0} \rightarrow \mathrm{h}^{+} \mathrm{h}^{-}$decays and found to be consistent with zero. Searches for the rare decays $\mathrm{D}_{(s)}^{+} \rightarrow \pi^{+} \mu^{+} \mu^{-}$, $\mathrm{D}_{(s)}^{+} \rightarrow \pi^{-} \mu^{+} \mu^{+}$and $\mathrm{D}^{0} \rightarrow \mu^{+} \mu^{-}$have found no evidence of signal, but have set the best limits on branching fractions to date. Thus, while no evidence for physics beyond the Standard Model has yet been found, the LHCb experiment is firmly establishing itself as a world leader in high precision charm physics measurements.

\section{References}

1. A. Augusto Alves Jr et al., JINST 3 (2008).

2. R. Aaij et al., arXiv:1309.6534 (2013).

3. R. Aaij et al., arXiv:1310.7201 (2013).

4. R. Aaij et al., JHEP 1204, p. 129 (2012).

5. R. Aaij et al., LHCb-CONF-2013-003 (2013).

6. R. Aaij et al., Phys.Lett. B723, 33 (2013).

7. A. Paul, I. I. Bigi and S. Recksiegel, Phys.Rev. D83, p. 114006 (2011).

8. R. Aaij et al., Phys.Lett. B724, 203 (2013).

9. R. Aaij et al., Phys.Lett. B725, 15 (2013). 